\title{
Conformational models of APP processing by gamma secretase based on analysis of pathogenic mutations
}

\author{
Meewhi Kim ${ }^{1, *}$ and Ilya Bezprozvanny ${ }^{1,2,3 *}$ \\ 1 Department of Physiology, UT Southwestern Medical Center at Dallas, TX, 75390, USA \\ 2 Laboratory of Molecular Neurodegeneration, Peter the Great St Petersburg State Polytechnical University, \\ St. Petersburg, 195251, Russia \\ 3 Laboratory of Synaptic Biology, Southern Federal University, Rostov-on-Don, 344006, Russia \\ * Correspondence: Meewhi.Kim@ UTSouthwestern.edu; Ilya.Bezprozvanny@UTSouthwestern.edu
}

\begin{abstract}
Proteolytic processing of amyloid precursor protein (APP) plays a critical role in pathogenesis of Azheimer's disease (AD). Sequential cleavage of APP by $\beta$ and $\gamma$ secretases leads to generation of $\mathrm{A} \beta 40$ (non-amyloidogenic) and $\mathrm{A} \beta 42$ (amyloidogenic) peptides. Presenilin-1 (PS1) or presenilin-2 (PS2) pay a role of catalytic subunit of $\gamma$-secretase. Multiple familial AD (FAD) mutations in APP, PS1, or PS2 result in increased A $\beta 42: A \beta 40$ ratio and accumulation of toxic $A \beta 42$ oligomers and plaques in patient brains. In this study we performed molecular modeling of APP complex with $\gamma$-secretase and analyzed potential effects of FAD mutations in APP and PS1. We noticed that all FAD mutations in APP transmembrane domain are predicted to cause an increase in the local disorder of its secondary structure. Based on structural analysis of known $\gamma$-secretase structures we proposed that APP can form a complex with $\gamma$-secretase in 2 potential conformations - M1 and M2. In conformation M1 transmembrane domain of APP forms a contact with perimembrane domain that follows the transmembrane domain 6 (TM6) in PS1 structure. In conformation M2 transmembrane domain of APP forms a contact with transmembrane domain 7 (TM7) in PS1 structure. By analyzing effects of PS1-FAD mutations on local protein disorder index, we discovered that these mutations increase conformational flexibility of $\mathrm{M} 2$ and reduce conformational flexibility of M1. Based on these results we proposed that M2 conformation, but not M1 conformation, of $\gamma$ secretase complex with APP leads to amyloidogenic (A $\beta 42$-generating) processing of APP. Our model predicts that APP processing in M1 conformation is favored by a curved membranes, such as membranes of early endosomes. In contrast, APP processing in M2 conformation is likely to be favored by a relatively flat membranes such as membranes of late endosomes and plasma membrane. These predictions are consistent with published biochemical analysis of APP processing at different subcellular locations. Our results also suggest that specific inhibitors of A $\beta 42$ production could be potentially developed by selectively targeting M2 conformation of $\gamma$ secretase complex with APP.
\end{abstract}

Keywords: gamma-secretase, APP, Alzheimer's disease; modelling; protein disorder

\section{Introduction}

Alzheimer's disease (AD) is a major health problem for developed nations that so far resisted development of effective therapies. Amyloid hypothesis of AD indicates that accumulation of amyloidogenic $\mathrm{A} \beta 42$ peptide is a major driving force responsible for $\mathrm{AD}$ [1-4]. Both amyloidogenic $A \beta 42$ peptide and non-amyloidogenic $A \beta 40$ peptide are generated as a result of sequential proteolytic cleavage of amyloid precursor protein (APP) by $\beta$ - and $\gamma$-secretases $[5,6]$. Amyloid hypothesis postulates that increased ratio of $\mathrm{A} \beta 42: \mathrm{A} \beta 40$ levels is a key pathogenic event in $\mathrm{AD}$ [1-4]. $\gamma$-secretase is a multiprotein membrane complex composed of nicastrin, presenilin enhancer 2 (Pen-2), anterior pharynx defective 1 (Aph1) and presenilin [7-9]. In addition to APP, $\gamma$-secretases cleave various type 
I transmembrane proteins, including Notch receptor [10]. Most likely because of these additional substrates, pharmacological targeting of $\gamma$-secretase so far failed to yield effective therapy for AD $[11,12]$. Multiple clinical trials of $\gamma$-secretase inhibitors failed partly due to side effects resulting from inhibition of cleavage of Notch receptor and other $\gamma$-secretase substrates, such as for example trial of semagacestat (LY-450139) [13]. Thus, there is a significant effort by the industry to develop "Notch-sparing" $\gamma$-secretase inhibitors that selectively block generation of $\mathrm{A} \beta 42$ but do not affect Notch receptor cleavage. Examples of such Notch-sparing compounds are avagacestat (BMS-708163) [14] and begacestat (GSI-953) [15]. However, avagacestat also failed in AD clinical studies [16, 17] and begacestat has not moved past phase 1 trial in clinical development [18]. Moreover, Notchsparing selectivity of avagacestat was questioned [19].

To inform this important direction of research, we here performed molecular modeling of APP complex with $\gamma$-secretase. In our modeling we relied on $\gamma$-secretase structures that have been recently solved by application of cryo-EM [20, 21]. Familial AD (FAD) mutations in APP or presenilins are known to have significant effects on production of $\mathrm{A} \beta 42$ and $\mathrm{A} \beta 40$ peptides and $\mathrm{A} \beta 42$ :A $\beta 40$ ratio [22-25]. We analyzed potential effects of these mutations on a conformational flexibility of APP complex with $\gamma$-secretase. Based on this analysis we proposed existence of 2 alternative conformations of APP complex with $\gamma$-secretase - M1 and M2. Furthermore, our analysis suggests that APP cleavage in M1 conformation favors A $\beta 40$ production, whereas APP in M2 conformation favors A $\beta 42$ production. Structural modeling also suggested that APP processing in M1 conformation is favored by a curved membranes, such as membranes of early endosomes. In contrast, APP processing in M2 conformation is likely to be favored by a relatively flat memranes such as membranes of late endosomes and plasma membrane. These predictions are consistent with biochemical analysis of APP processing in different subcellular locations [26-31]. Proposed co-existence of M1 and M2 complexes of APP and $\gamma$-secretase may help structure-functional analysis of APP processing and may potentially facilitate development of more selective Notch-sparing inhibitors of $\gamma$-secretase for therapeutic use.

\section{Results}

\subsection{AD mutations destabilize secondary structure of APP transmembrane domain}

APP is a 770 amino-acid protein with a single transmembrane domain in the carboxyterminal end (Fig 1A). In the process of generating A $\beta$ peptides, APP is cleaved initially by a $\beta$-secretase at position 671 and then by a $\gamma$-secretase within a transmembrane domain (Fig 1A). There is a number of AD-causing mutations in APP sequence, most of them located within APP transmembrane domain (Fig 1A) [22-25]. In order to understand potential impact of these mutations on APP secondary structure, we calculated changes in local disorder index (DI) resulting from these mutations using web based program (see Methods for details). For this calculation we utilized a fragment of APP sequence (671730) that starts at the $\beta$-secretase cleavage site and ends several amino acids after the transmembrane domain (Fig 1A). In our calculations, this fragment was modeled as a continuous $\alpha$-helix. The AD-causing mutations in transmembrane domain of APP are located between amino acids 705 and 723 (Fig 1A). In some of these positions only a single substitution (MUT1) is known to be pathogenic, but in some positions several pathogenic substitutions have been reported. In these positions we performed calculations for one more potential substitution (MUT2). The AD-causing mutations that we analyzed were as follows - L705V, A713V, T714A (Iranian), T714I (Austrian), V715M (French), V715A (German), I716V (Florida), I716F (Iberian), V717F (Indiana), V717I (London), T719N, T719P, M722K, and L723P (Australian) [22-25]. This analysis revealed that all AD-causing mutations in the transmembrane domain of APP significantly destabilize the secondary structure of the APP domain, with the changes in the local disorder index in the range 
between 10-12 for the most mutations (Fig 1B). Based on these results we concluded that destabilization and partial unwinding of $\alpha$-helical structure of APP transmembrane domain facilitates amyloidogenic processing of APP by $\gamma$-secretase and generation of A $\beta 42$ peptide.

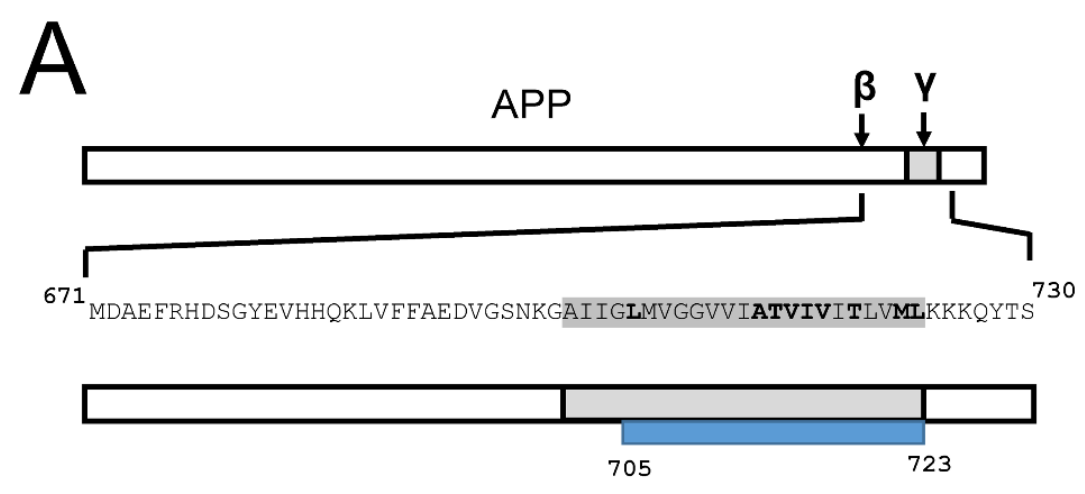

B
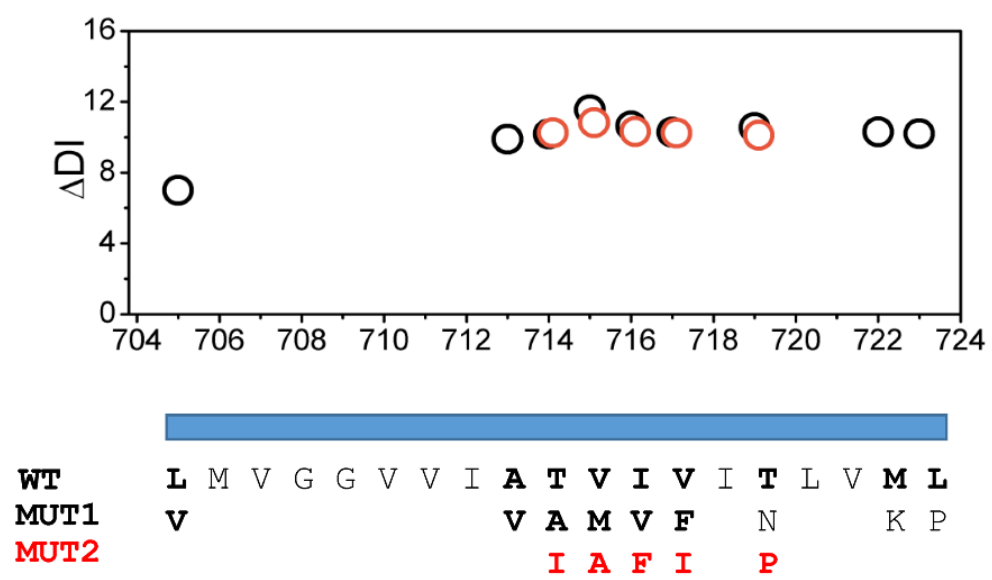

Figure 1. FAD mutations of APP transmembrane domain destabilize the secondary structure. A, Domain structure of APP with locations of $\beta$ - and $\gamma$-secretase cleavage sites indicated. The sequence of APP from $\beta$-secretase cleavage site (M671) to soluble region following transmembrane domain (S730) is indicated. Transmembrane domain is shown in grey, positions that mutated in FAD are shown by bold font. Blue bar shows boundaries of the mutated region. B, Calculated difference in disorder index $(\triangle \mathrm{DI})$ for FAD mutants is shown for Mut1 (open circles) and Mut2 (red circles)

\subsection{Protein Structural models of APP complex with $\gamma$-secretase}

To better understand mechanistic basis of these results, we generated a structural model of APP complex with $\gamma$-secretase using $\gamma$-secretase structures that have been recently solved by application of cryo-EM [20, 21]. Presenilin-1 (PS1) or presenilin-2 (PS2) are catalytic subunits of $\gamma$-secretase [7-9]. In our modeling we focused on PS1. The matured PS1 structures within $\gamma$-secretase are heterodimers of PS1-NT (TM1-TM6) and PS1-CT (TM7-TM9) fragments. We downloaded all $\gamma$-secretase structures from protein data bank (PDB 6IDF, 6IYC, 5A63, 5FN2,5FN3, 5FN4, 5FN5, 4UIS) and closely examined conformation of $\gamma$-secretase active site formed by TM6 and TM7 transmembrane domains of PS1. The reported PS1 structures miss most of the soluble domains, with the exception of a small portion of a perimembrane region that follows TM6. The structure of this domain is solved in different conformations ranging from unstructured conformation to a loop and $\alpha$-helix near membrane [20, 21] 
We found that three TM helixes of PS1-CT (TM7-TM9) are largely overlapping in all structures but TM helixes of PS1-NT (TM1-TM6) adopt different conformations in different structures (Fig 2A compares structures from 5FN4, 5FN5, and 6IYC). In particular, TM6 helix is found in two different conformations as shown on Fig 2A. We used these structures to model possible locations of APP transmembrane domain $\alpha$ helix within an active site of a $\gamma$-secretase. As a result of the model building, we were able to produce 2 possible protein models of the complex - model 1 (M1, based on PDB 6IYC) (Fig 2B) and model 2 (M2, based on 5FN3) (Fig 2C). In designing these models we made sure that transmembrane domain of APP is in direct contact with $\gamma$-secretase active site formed by D257 (TM6) and D385 (TM7) residues of PS1. In model M1 APP forms a contact with perimembrane domain that follows TM6 in PS1 structure (based on PDB 6IYC) (Fig 2B). In model M2 APP forms a contact with TM7 domains (based on PDB 5FN3) (Fig 2C). Both models were optimized to satisfy overall protein-protein interactions from polar, nonpolar and aromatic residues (see Methods for details).
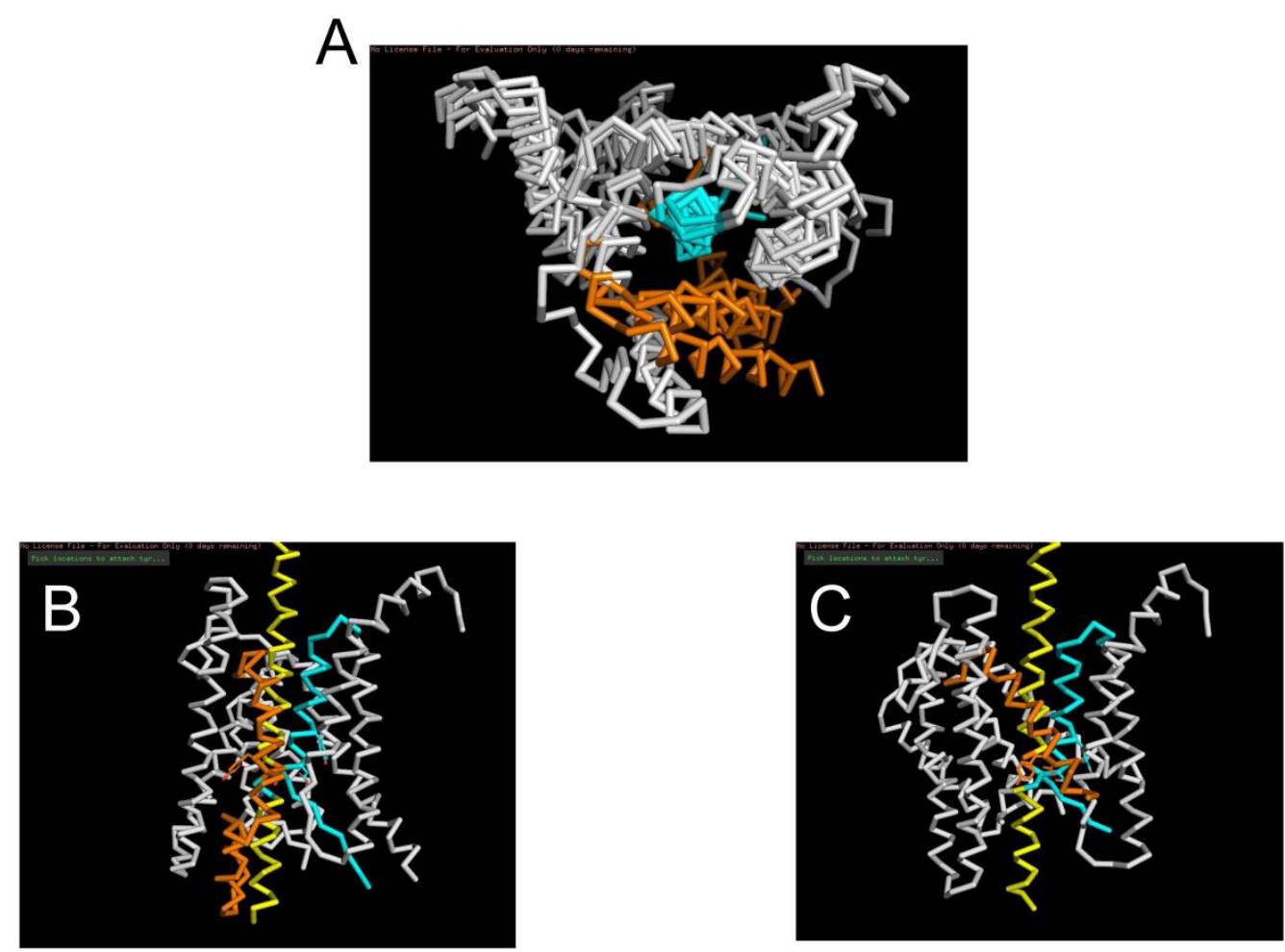

Figure 2. Structural models of $\gamma$-secretase/APP complex.

A. An overlay of PS1 structures (PDB 5FN4, 5FN5, 6IYC). TM7 is colored in cyanate and TM6 is colored in orange. All other TMs are colored in grey.

B. M1 model of $\gamma$-secretase complex with APP. D1-TM6 region is colored in orange, TM7 is colored in cyanate and APP is colored in yellow.

C. M2 model of $\gamma$-secretase complex with APP. TM6 region is colored in orange, D2-TM7 is colored in cyanate and APP is colored in yellow 


\subsection{Effects of AD mutations in PS1 on stability of APP complex with $\gamma$-secretase}

PS1 contains 9 TMs (Fig 3A) with AD-causing mutations spread across all of them [23, 25]. Structural modeling suggests that TM6 and TM7 form direct contact with APP transmemrabe domain (Fig 2). What is the influence of the mutations within TM6 and TM7 domain on association between APP and $\gamma$-secretase? To answer this question, we calculated the changes in local disorder index (DI) resulting from PS1 mutations by using the same approach as described above for APP transmembrane domain (see Methods for details). For this analysis we selected a region between A251 and A400 of PS1 (Fig 3A, mutations shown in bold font). This region is directly involved in molecular interactions with APP (Fig 2) and includes a part TM6 domain, the large soluble region between TM6 and TM7 domains that contains endoproteolysis cleavage site (at position L286), and TM7 domain (Fig 3A). D257 and D385 aspartates that form catalytic site of $\gamma$-secretase are both located within this region (Fig 3A). In our analysis we excluded a large portion of soluble loop between TM6 and TM7 domains (positions between 287-363) as secondary structure of this region is not resolved by cryoEM and it is not involved in significant interactions with APP based on our model (Fig 2). To separate the effects of the mutations in TM6 and TM7 domains in our calculations, we divided relevant PS1 sequence into 2 regions D1 and D2 (Fig 3A). D1 region included mutations in positions Y256-A285 and D2 region included mutations in positions S365-A396 (Fig 3A). To estimate the change in local disorder index additional flanking transmembrane sequences from TM6 and TM7 were also included in the calculations (Fig 3A). The results of these calculations for the representative AD-causing mutations in the region between Y256 and A396 are shown on Fig 3B. Similar results were obtained when other pathogenic mutations of PS1 in the same positions were evaluated (data not shown). It is noticeable that most AD-causing mutations in D1 region resulted in negative change in DI, consistent with reduction in conformational flexibility of local PS1 structure (Fig 3B). The exception to this rule are mutations A260V and $\mathrm{V} 261 \mathrm{~F}$ which resulted in positive change in $\mathrm{DI}$ (Fig 3B). In contrast, most $\mathrm{AD}$-causing mutations in D2 region resulted in positive change in DI, consistent with increased conformational flexibility of PS1 structure (Fig 3B). The exceptions to the later rule are mutations G378V, G384A and Y389F that resulted in negative change in DI (Fig 3B).

It is interesting to compare these results with structural modeling of APP complex with $\gamma$-secretase (Fig 2). The PS1 region corresponding to D1 is involved in direct association with transmembrane domain of APP according to the M1 model of the complex (Fig 2B) but not according to the M2 model (Fig 2C). In contrast, the PS1 region corresponding to D2 is involved in association with transmembrane domain of APP according to the M2 model of the complex (Fig 2C) but not according to the M1 model (Fig 2B). Therefore, it appears that most disease-causing mutations reduce conformational flexibility of $\gamma$-secretase complex with APP in M1 conformation but increase conformational flexibility of $\gamma$-secretase complex with APP in M2 conformation. Our previous analysis of ADcausing mutations in APP transmembrane domain revealed that all of these mutations increased disorder index and conformational flexibility of APP (Fig 1B). To explain these findings, we propose that PS1 mutations in D2 region act similar to APP mutations by promoting conformational flexibility of the complex in M2 conformation, resulting in increased generation of A $\beta 42$. In contrast, we reason that PS1 mutations in D1 region act by reducing conformation flexibility of the complex in M1 configuration, resulting in impaired production of $A \beta 40$. An overall result of all these mutations (in APP, and in D1 and D2 regions of PS1) is increased ratio of A $\beta 42: A \beta 40$ produced by the $\gamma$-secretase. An interesting exception to this rule are 5 mutations in D1 and D2 regions discussed above. It is striking that 4 out of these 5 mutations are located within immediate proximity of catalytic aspartates D257 and D385 in the secondary structure of PS1 (Figs 3C, 3D). Thus, most likely these 4 mutations have direct impact on the catalytic reaction mediated by D257 and D385, in contrast to other mutations in D1 and D2 regions that most likely act by affecting overall $\gamma$-secretase movement during APP proteolysis. 
A
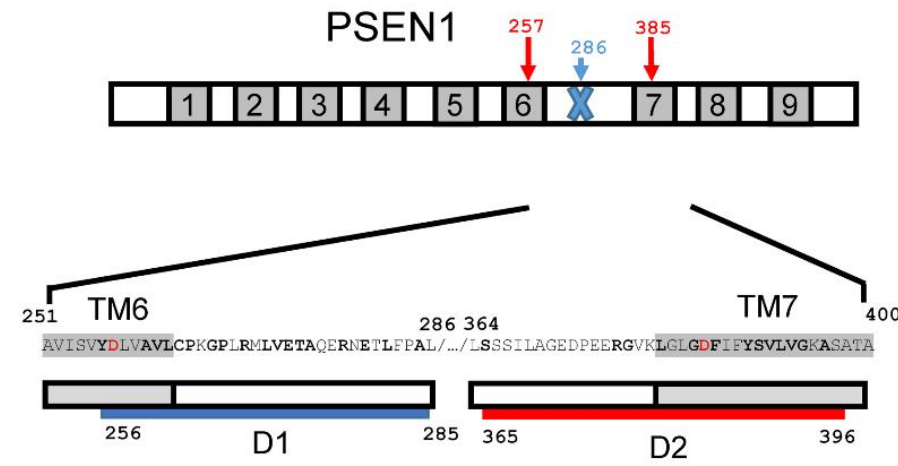

B

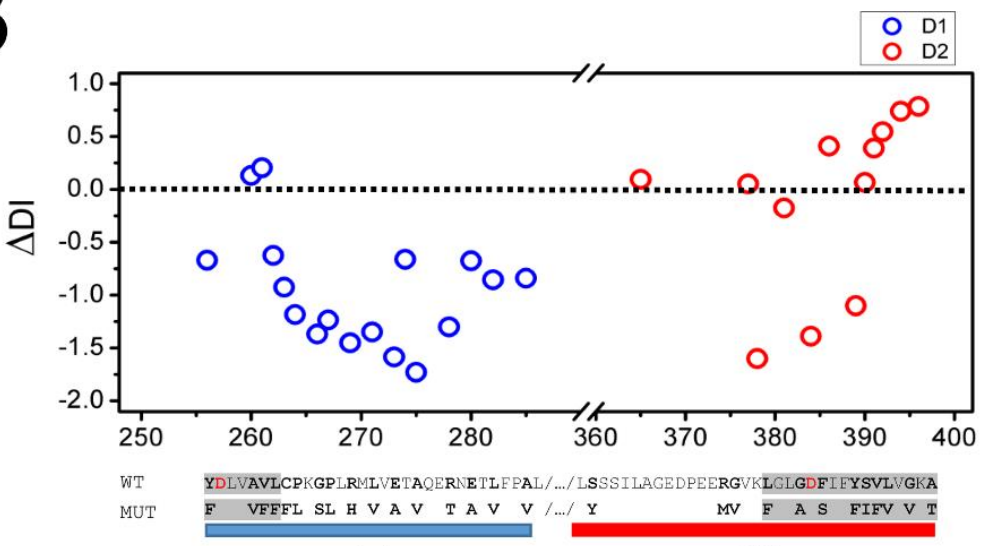

D1

D2

C
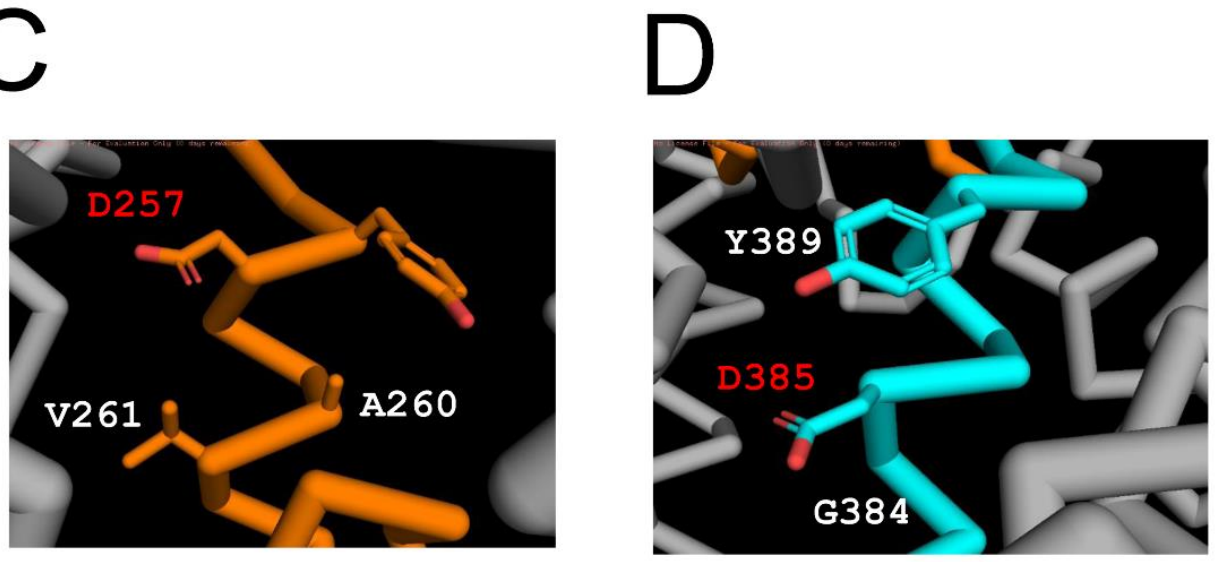

Figure 3. Effects of FAD mutations in PS1 on stability of APP complex with $\gamma$-secretase..

A. Bar diagram shows the domain structur of PS1 with locations of catalytic aspartates (D257 and D385) and location of endoproteolytic cleavage site (L286) indicated. The sequences of D1 region (A251-L286) and D2 region (L364-A400) are indicated. Transmembrane domains are shown in grey, positions that mutated in FAD are shown by bold font, catalytic aspartates are shown by red font. Blue bar (D1) and red bar (D2) shows boundaries of the mutated region that was analyzed.

B. Calculated difference in disorder index $(\triangle \mathrm{DI})$ for FAD mutants is shown for D1 region (blue circles) and D2 region (red circles).

C. High resolution structural model of $\gamma$-secretase active site with positions of V261 and A260 shown relative to the catalytic aspartate D257

D. High resolution structural model of $\gamma$-secretase active site with positions of Y389 and G384 shown relative to the catalytic aspartate D285

\subsection{Effects of membrane curvature on APP processing by $\gamma$-secretase.}

Processing of APP by $\gamma$-secretase occurs in plasma membrane and in early and late endosomal compartments [26-30]. Plasma membrane is relatively flat but endosomal 
membranes are curved. Because of the difference in size, membranes of early endosomes (typical diameter from $40 \mathrm{~nm}$ to $100 \mathrm{~nm}$ ) are significantly more curved than membranes of larger (typical diameter from $250 \mathrm{~nm}$ to $400 \mathrm{~nm}$ ) later endosomes [31]. What effect, if any, can the curvature of the membrane have on APP processing by $\gamma$-secretase? To answer this question, we modeled APP $/ \gamma$-secretase complex in M1 and M2 conformations in the membranes with different curvature. Based on known cryo-EM APP $/ \gamma$ secretase complex structure [21], in the first approximation we modelled PS1 embedded in the flat plasma membrane as a cylinder with diameter of $0.40 \mathrm{~nm}$ and height $0.45 \mathrm{~nm}$ (average size measured from PS structures of PDBs) (Fig 4A). We further reasoned that as a result of membrane expansion the shape of PS1 embedded into the curved membrane would be affected by an expansion on the cytosolic side by a distance equal to $\mathrm{d}$. From our estimates this distance for early endosomes (d1) is in the range between $4 \AA$ and $12 \AA$, depending on the size of the endosome (Fig $4 \mathrm{~B})$. For late endosomes this distance (d2) is estimated to be less than $1 \AA$ (Fig 4C). We further reasoned that these differences in PS1 geometry may have differential effects on APP processing by $\gamma$-secretase in M1 and M2 conformations.

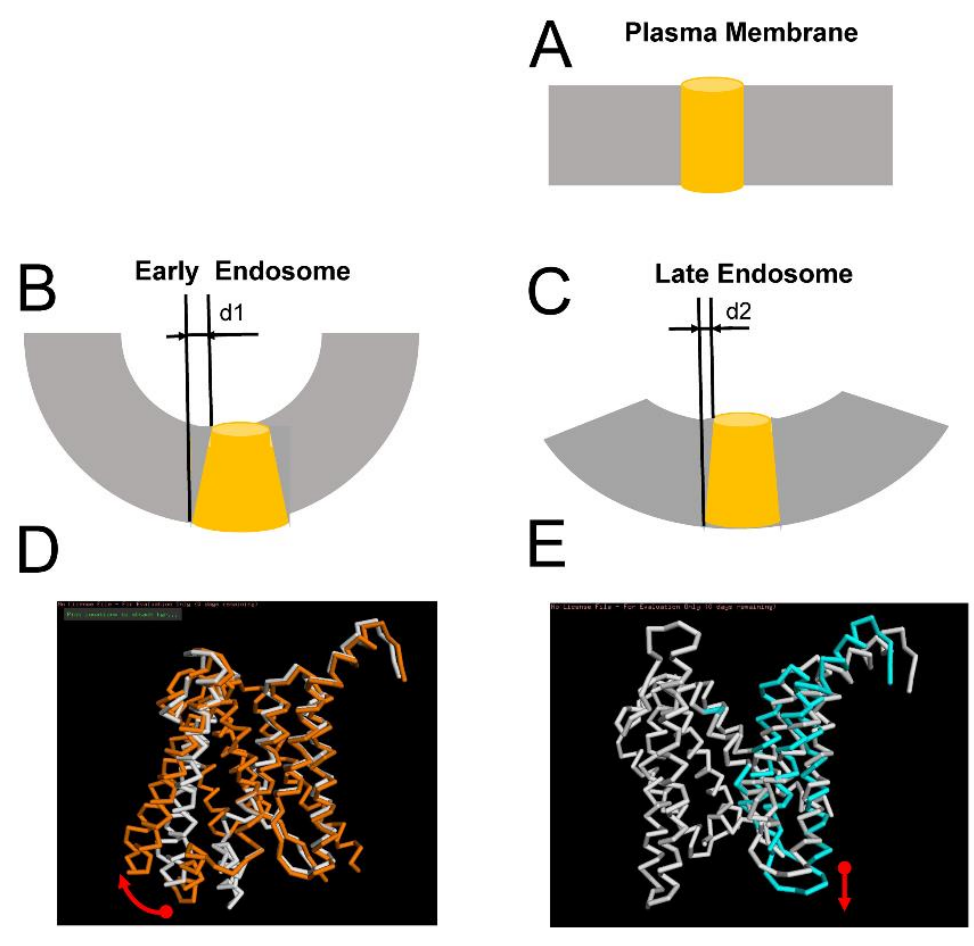

Figure 4. Effects of membrane curvature on $\gamma$-secretase activity.

A. PS1 is modelled as a cylinder shape in the plasma membrane

B, C PS1 in modelled as a truncated cone shape in the curved membrane of early (B) and late (C) endosomes. Expansion of PS1 shape at the cytosolic surface of the membrane is shown as d1 (B) and $\mathrm{d} 2(\mathrm{C})$.

D. APP processing by $\gamma$-secretase is modeled as lateral movement of PS1 in M1 conformation (shown as an overlay). The movement is a $7 \AA ̊$ lateral movement between known PS1 structure 6IYC (white) and PS1 conformation in model M1 (orange).

E. APP processing by $\gamma$-secretase is modeled as stretching movement of PS1 in M2 conformation (shown as an overlay). The movement is $3 \AA ̊$ expansion of TM6 domain between known PS1 structure 5FN3 (white) and PS1 conformation in model M2 (cyanate)

Structural modeling suggests that peri-membrane region D1 that follows TM6 region of PS1 is involved in direct association with APP transmembane domain in M1 conformation of APP $/ \gamma$-secretase complex (Fig 2B). Our hypothesis is that APP cleavage by $\gamma$ secretase in M1 conformation involves rotation of TM6 and D1 regions (Fig 4D) and such 
movement should be facilitated when PS1 is embedded in the curved membrane with expanded cytosolic interface. In contrast, inner membrane region D2 of PS1 TM7 is involved in direct association with transmembrane domain of APP in M2 conformation of $\gamma$-secretase complex (Fig 2C). We propose that APP cleavage by $\gamma$-secretase in M2 conformation involves stretching of TM7 region perpendicular to the membrane surface (Fig 4E) and that such movement is facilitated when PS1 is embedded into flat membranes or into the membranes with low curvature. Based on this analysis, we suggest that APP cleavage by $\gamma$-secretase in early endosomal compartments is biased towards M1-mediated processing and A $\beta 40$ production, while APP cleavage by $\gamma$-secretase in late endosome compartments and in the plasma membrane is biased towards M2-mediated processing and A 342 production. This hypothesis is consistent with biochemical analysis of APP processing in different subcellular locations [26-30].

\section{Discussion}

\subsection{Comparison of M1 and M2 models with known structures of APP/ $\gamma$-secretase complex.}

The structure of APP $/ \gamma$-secretase complex was previously solved by cryo-EM (PDB 6IYC) [21]. This structure was critical for building model M1 (Fig 2B). In this structure the transmembrane domain of APP peptide (L705-K726) forms molecular interactions with TM2, TM3, TM5, TM6 and TM7 of PS1. The secondary structure of the APP protein in structure 6IYC make a transition from $\alpha$ to $\beta$ conformation at carboxy terminal end, where it forms an antiparallel $\beta$ - strand interaction with $\beta$-strand of TM7 of PS1. Similar to this structure, our models also indicate that APP substrate accesses $\gamma$-secretase active site by interacting with TM5, TM6, and TM7 of PS1 (Figs 2B and 2C). However, our models make different predictions regarding conformational movements of $\gamma$-secretase during catalytic reaction of APP hydrolysis. Cryo-EM structure (PDB 6IYC) suggests that catalytic reaction involves interaction of $\alpha$-helical amino-terminal APP region with TM2 and TM3 of PS1 and $\beta$-strand association of carboxy-terminal APP region with TM7 [21]. In contrast, we modeled association of APP $\alpha$-helix with D1-TM6 (in M1 conformation, Fig 2B) or with D2-TM7 (in M2 conformation, Fig 2C). Based on these structural models we predicted that APP cleavage by $\gamma$-secretase in M1 conformation involves rotation of TM6 and D1 regions (Fig 4D) and that APP cleavage by $\gamma$-secretase in M2 conformation involves stretching of TM7 region perpendicular to the membrane surface (Fig 4E). This hypothesis is consistent with biochemical analysis of APP processing in different subcellular locations [26-30]. It should be noted that our analysis was primarily focused on the catalytic site of $\gamma$-secretase that is composed by TM6 and TM7 domains of PS1. However, many FAD mutations in presenilins are located outside of this region [23, 25] and it is possible that mutations in these additional domains also affect conformational flexibility of $\gamma$-secretase catalytic site. We have not been able to analyze effects of these mutations as our analysis is limited to calculations of local protein disorder and not applicable for analysis of long-range effects. More sophisticated modeling and molecular dynamics simulation approaches may help to address this question by taking an advantage of known structures of $\gamma$-secretase [20, 21].

\subsection{Potential implications for development of selective $\gamma$-secretase inhibitors}

Amyloid hypothesis predicts that $\gamma$-secretase should be most promising therapeutic target for treatment of $\mathrm{AD}$ as inhibition of $\mathrm{A} \beta 42$ production should stop progression of AD pathology [1-4]. However, $\gamma$-secretase inhibitors such as for example semagacestat (LY-450139) failed in clinical trails [11, 12]. One of potential reasons for these failures are side effects resulting from inhibition of cleavage of Notch receptor and other $\gamma$-secretase substrates. To minimize these problems industry has been focused on developing "Notch-sparing" $\gamma$-secretase inhibitors that selectively block generation of A $\beta 42$ but do not affect Notch receptor cleavage. However, such inhibitors as for example avagacestat (BMS-708163) also failed in clinical trials [16, 17]. These efforts could be informed by re- 
sults of our modeling studies. Our hypothesis suggest that specific inhibitors of M2 conformation of $\gamma$-secretase/APP complex may selectively inhibit production of A $\beta 42$. Such selective inhibitors may be potentially developed by structure-guided virtual screening and selection of small molecules that more potently inhibit $\gamma$-secretase in M2 conformation (Fig 2C) than in M1 conformation (Fig 2B).

\section{Materials and Methods}

\subsection{Calculations of disorder index of FAD mutations in APP and PS1}

To calculate changes in local disorder index (DI) we used web based program DISOPRED3 [32] available at http://bioinf.cs.ucl.ac.uk/psipred/ using a fragment of APP sequence (M671-S730). The AD-causing mutations that we analyzed were as follows L705V, A713V, T714A (Iranian), T714I (Austrian), V715M (French), V715A (German), I716V (Florida), I716F (Iberian), V717F (Indiana), V717I (London), T719N, T719P, M722K, and L723P (Australian). Wild type and single residue mutated fragments of APP were subjected to DI calculation by DISOPRED3 and the calculated DI for each mutant was subtracted from wild type DI to obtain $\triangle \mathrm{DI}$.

Similar approach was used in analysis of PS1 FAD using D1 (A251-L286) and D2 (L364-A400) regions in calculations. To calculate $\triangle \mathrm{DI}$ DI was calculated for wild type D1 and D2 sequence and for a series of FAD mutations in D1 (Y256F, A260V, V261F, L262F, C263F, P264L, G266S, P267T, R269H, L271V, E273A, T274R, A275V, R278T, E280A, L282V, A285V) and D2 (S365Y, L381F, Y389F, R377M, G378V, G384A, F386S, S390I, V391F, L392V, G394V, A396T) to yield $\Delta$ DI for each mutation.

\subsection{Structural models of APP complex with $\gamma$-secretase}

M1 model of APP complex with $\gamma$-secretase is based on PDB 6IYC [21] and M2 model of APP complex with $\gamma$-secretase is based on PDB 5FN3 [20] Transmembrane domain of APP was modelled as an $\alpha$-helix. At the first step we modelled molecular interactions with APP transmembrane domain with TM5, TM6 and TM7 in PS structures. Because 6IYC structure was solved in the complex with APP, only slight adjustment of the structure was required to generate model M1. To generate M2 model, PS1-NT structure from 5FN3 was adjusted by rotating further using loop regions without disturbing the active site formed by D257 and D385. Such manipulation is similar to sliding movement observed for PS1-NT in known structures [PDB 6idf, 6iyc, 5a63, 5fn2,5fn3, 5fn4, 5fn5, 4uis, 4hyg, 4hyc, 4hyd]. At the second step APP in model M1 was modeled to form a contact with perimembrane domain D1 that follows TM6 in PS1 structure. APP in model M2 was modeled to form contact with TM7 domain in PS1 structure. On the third step for both models we placed VIAT sequence of APP at the $\gamma$-secretase catalytic site formed by D257 and D385. APP $\alpha$-helix was adjusted to have proper interactions with polar, nonpolar and aromatic residues within TM5, TM6, and TM7 domains to yeld final version of M1 and M2 models of the complex. While tuning these interactions VIAT residues remained at the active site. 
Supplementary Materials: None. The following are available online at www.mdpi.com/xxx/s1,

Author Contributions: Conceptualization, MWK and IB; methodology, MWK; formal analysis, MWK and IB; investigation, MWK and IB; resources, IB; data curation, MWK and IB. writingoriginal draft preparation, MWK and IB; writing - review and editing, MWK and IB; visualization, MWK and IB; supervision, IB; project administration, IB; funding acquisition, IB. All authors have read and agreed to the published version of the manuscript

Funding: This research was funded by the Russian Science Foundation Grant 19-15-00184 (I.B.) and by the National Institutes of Health grants R56AG071310 (I.B.) and R01AG055577 (I.B.). I.B. holds the Carl J. and Hortense M. Thomsen Chair in Alzheimer's Disease Research.

Institutional Review Board Statement: Not applicable.

Informed Consent Statement: Not applicable.

Data Availability Statement: Not applicable.

Acknowledgments: We are thankful to members of Bezprozvanny laboratory for useful discussions and to Dr Xiaochen Bai for comments on the manuscript..

Conflicts of Interest: The authors declare no conflict of interest.

\section{References}

1. Selkoe, D. J., Alzheimer's disease is a synaptic failure. Science 2002, 298, (5594), 789-91.

2. Selkoe, D. J.; Hardy, J., The amyloid hypothesis of Alzheimer's disease at 25 years. EMBO Mol Med 2016, 8, (6), 595-608.

3. Hardy, J.; Selkoe, D. J., The amyloid hypothesis of Alzheimer's disease: progress and problems on the road to therapeutics. Science 2002, 297, (5580), 353-6.

4. Hardy, J., The amyloid hypothesis for Alzheimer's disease: a critical reappraisal. J Neurochem 2009, 110, (4), $1129-34$.

5. O'Brien, R. J.; Wong, P. C., Amyloid precursor protein processing and Alzheimer's disease. Annu Rev Neurosci 2011, 34, 185204.

6. Hampel, H.; Hardy, J.; Blennow, K.; Chen, C.; Perry, G.; Kim, S. H.; Villemagne, V. L.; Aisen, P.; Vendruscolo, M.; Iwatsubo, T.; Masters, C. L.; Cho, M.; Lannfelt, L.; Cummings, J. L.; Vergallo, A., The Amyloid-beta Pathway in Alzheimer's Disease. Mol Psychiatry 2021.

7. De Strooper, B., Aph-1, Pen-2, and Nicastrin with Presenilin generate an active gamma-Secretase complex. Neuron 2003, 38, (1), 9-12.

8. Tolia, A.; De Strooper, B., Structure and function of gamma-secretase. Semin Cell Dev Biol 2009, 20, (2), 211-8.

9. Bergmans, B. A.; De Strooper, B., gamma-secretases: from cell biology to therapeutic strategies. Lancet Neurol 2010, 9, (2), 215-226.

10. Kopan, R.; Ilagan, M. X., Gamma-secretase: proteasome of the membrane? Nat Rev Mol Cell Biol 2004, 5, (6), 499-504.

11. Karran, E.; Hardy, J., A critique of the drug discovery and phase 3 clinical programs targeting the amyloid hypothesis for Alzheimer disease. Ann Neurol 2014, 76, (2), 185-205.

12. De Strooper, B., Lessons from a failed gamma-secretase Alzheimer trial. Cell 2014, 159, (4), 721-6.

13. Doody, R. S.; Raman, R.; Farlow, M.; Iwatsubo, T.; Vellas, B.; Joffe, S.; Kieburtz, K.; He, F.; Sun, X.; Thomas, R. G.; Aisen, P. S.; Alzheimer's Disease Cooperative Study Steering, C.; Siemers, E.; Sethuraman, G.; Mohs, R.; Semagacestat Study, G., A phase 3 trial of semagacestat for treatment of Alzheimer's disease. N Engl J Med 2013, 369, (4), 341-50.

14. Albright, C. F.; Dockens, R. C.; Meredith, J. E., Jr.; Olson, R. E.; Slemmon, R.; Lentz, K. A.; Wang, J. S.; Denton, R. R.; Pilcher, G.; Rhyne, P. W.; Raybon, J. J.; Barten, D. M.; Burton, C.; Toyn, J. H.; Sankaranarayanan, S.; Polson, C.; Guss, V.; White, R.; Simutis, F.; Sanderson, T.; Gillman, K. W.; Starrett, J. E., Jr.; Bronson, J.; Sverdlov, O.; Huang, S. P.; Castaneda, L.; Feldman, H.; Coric, V.; Zaczek, R.; Macor, J. E.; Houston, J.; Berman, R. M.; Tong, G., Pharmacodynamics of selective inhibition of gamma-secretase by avagacestat. J Pharmacol Exp Ther 2013, 344, (3), 686-95. 
15. Mayer, S. C.; Kreft, A. F.; Harrison, B.; Abou-Gharbia, M.; Antane, M.; Aschmies, S.; Atchison, K.; Chlenov, M.; Cole, D. C.; Comery, T.; Diamantidis, G.; Ellingboe, J.; Fan, K.; Galante, R.; Gonzales, C.; Ho, D. M.; Hoke, M. E.; Hu, Y.; Huryn, D.; Jain, U.; Jin, M.; Kremer, K.; Kubrak, D.; Lin, M.; Lu, P.; Magolda, R.; Martone, R.; Moore, W.; Oganesian, A.; Pangalos, M. N.; Porte, A.; Reinhart, P.; Resnick, L.; Riddell, D. R.; Sonnenberg-Reines, J.; Stock, J. R.; Sun, S. C.; Wagner, E.; Wang, T.; Woller, K.; Xu, Z.; Zaleska, M. M.; Zeldis, J.; Zhang, M.; Zhou, H.; Jacobsen, J. S., Discovery of begacestat, a Notch-1-sparing gammasecretase inhibitor for the treatment of Alzheimer's disease. J Med Chem 2008, 51, (23), 7348-51.

16. Coric, V.; van Dyck, C. H.; Salloway, S.; Andreasen, N.; Brody, M.; Richter, R. W.; Soininen, H.; Thein, S.; Shiovitz, T.; Pilcher, G.; Colby, S.; Rollin, L.; Dockens, R.; Pachai, C.; Portelius, E.; Andreasson, U.; Blennow, K.; Soares, H.; Albright, C.; Feldman, H. H.; Berman, R. M., Safety and tolerability of the gamma-secretase inhibitor avagacestat in a phase 2 study of mild to moderate Alzheimer disease. Arch Neurol 2012, 69, (11), 1430-40.

17. Dockens, R.; Wang, J. S.; Castaneda, L.; Sverdlov, O.; Huang, S. P.; Slemmon, R.; Gu, H.; Wong, O.; Li, H.; Berman, R. M.; Smith, C.; Albright, C. F.; Tong, G., A placebo-controlled, multiple ascending dose study to evaluate the safety, pharmacokinetics and pharmacodynamics of avagacestat (BMS-708163) in healthy young and elderly subjects. Clin Pharmacokinet 2012, 51, (10), 681-93.

18. Hopkins, C. R., ACS chemical neuroscience molecule spotlight on Begacestat (GSI-953). ACS Chem Neurosci 2012,3 , (1), 3-4.

19. Crump, C. J.; Castro, S. V.; Wang, F.; Pozdnyakov, N.; Ballard, T. E.; Sisodia, S. S.; Bales, K. R.; Johnson, D. S.; Li, Y. M., BMS708,163 targets presenilin and lacks notch-sparing activity. Biochemistry 2012, 51, (37), 7209-11.

20. Bai, X. C.; Yan, C.; Yang, G.; Lu, P.; Ma, D.; Sun, L.; Zhou, R.; Scheres, S. H.; Shi, Y., An atomic structure of human gammasecretase. Nature 2015, 525, (7568), 212-7.

21. Zhou, R.; Yang, G.; Guo, X.; Zhou, Q.; Lei, J.; Shi, Y., Recognition of the amyloid precursor protein by human gammasecretase. Science 2019, 363, (6428).

Tcw, J.; Goate, A. M., Genetics of beta-Amyloid Precursor Protein in Alzheimer's Disease. Cold Spring Harb Perspect Med 2017, 7, (6).

23. Cacace, R.; Sleegers, K.; Van Broeckhoven, C., Molecular genetics of early-onset Alzheimer's disease revisited. Alzheimers Dement 2016, 12, (6), 733-48.

24. Neuner, S. M.; Tcw, J.; Goate, A. M., Genetic architecture of Alzheimer's disease. Neurobiol Dis 2020, $143,104976$.

25. Bekris, L. M.; Yu, C. E.; Bird, T. D.; Tsuang, D. W., Genetics of Alzheimer disease. J Geriatr Psychiatry Neurol 2010, 23, (4), 213-27.

26. Kanatsu, K.; Tomita, T., Membrane trafficking and proteolytic activity of gamma-secretase in Alzheimer's disease. Biol Chem 2016, 397, (9), 827-35.

Sannerud, R.; Esselens, C.; Ejsmont, P.; Mattera, R.; Rochin, L.; Tharkeshwar, A. K.; De Baets, G.; De Wever, V.; Habets, R.; Baert, V.; Vermeire, W.; Michiels, C.; Groot, A. J.; Wouters, R.; Dillen, K.; Vints, K.; Baatsen, P.; Munck, S.; Derua, R.; Waelkens, E.; Basi, G. S.; Mercken, M.; Vooijs, M.; Bollen, M.; Schymkowitz, J.; Rousseau, F.; Bonifacino, J. S.; Van Niel, G.; De Strooper, B.; Annaert, W., Restricted Location of PSEN2/gamma-Secretase Determines Substrate Specificity and Generates an Intracellular Abeta Pool. Cell 2016, 166, (1), 193-208.

28. Ubelmann, F.; Burrinha, T.; Salavessa, L.; Gomes, R.; Ferreira, C.; Moreno, N.; Guimas Almeida, C., Bin1 and CD2AP polarise the endocytic generation of beta-amyloid. EMBO Rep 2017, 18, (1), 102-122.

29. Ahn, K.; Shelton, C. C.; Tian, Y.; Zhang, X.; Gilchrist, M. L.; Sisodia, S. S.; Li, Y. M., Activation and intrinsic gamma-secretase activity of presenilin 1. Proc Natl Acad Sci U S A 2010, 107, (50), 21435-40.

30. Schedin-Weiss, S.; Caesar, I.; Winblad, B.; Blom, H.; Tjernberg, L. O., Super-resolution microscopy reveals gamma-secretase at both sides of the neuronal synapse. Acta Neuropathol Commun 2016, 4, 29.

31. Cooney, J. R.; Hurlburt, J. L.; Selig, D. K.; Harris, K. M.; Fiala, J. C., Endosomal compartments serve multiple hippocampal dendritic spines from a widespread rather than a local store of recycling membrane. J Neurosci 2002, 22, (6), $2215-24$. 
32. Jones, D. T.; Cozzetto, D., DISOPRED3: precise disordered region predictions with annotated protein-binding activity. Bioinformatics 2014, 31, (6), 857-863. 